\title{
Adaptive Tracking Control of an Uncertain Nonholonomic Robot*
}

\author{
Nan Hu, Chaoli Wang \\ School of Optical-Electrical and Computer Engineering, University of Shanghai \\ for Science and Technology, Shanghai, China \\ E-mail:hn19861215@126.com \\ Received September 3, 2011; revised September 24, 2011; accepted October 19, 2011
}

\begin{abstract}
In this paper, a new controller is proposed by using backstepping method for the trajectory tracking problem of nonholonomic dynamic mobile robots with nonholonomic constraints under the condition that there is a distance between the mass center and the geometrical center and the distance is unknown. And an adaptive feedback controller is also proposed for the case that some kinematic parameters and dynamic parameters are uncertain. The asymptotical stability of the control system is proved with Lyapunov stability theory. The simulation results show the effectiveness of the proposed controller. The comparison with the previous methods is made to show the effectiveness of the method in this article.
\end{abstract}

Keywords: Nonholonomic Systems, Wheeled Mobile Robot, Adaptive Control, Tracking Control

\section{Introduction}

In recent years, the control problem of the nonholonomic systems has been widely investigated. The wheeled mobile robots have become a practical benchmark of these systems and the hot spot of research. However, it is proved that such systems cannot be stabilized by use smooth pure state feedback controllers because nonholonomic systems don't satisfy the Brockett's necessary condition of smooth feedback stabilization [1]. Therefore the researchers proposed many control methods to meet the challenge, for instance, continuous time-varying feedback control laws [2-4]; discontinuous feedback control laws [5-7]; hybrid feedback control laws [8]; and optimal control laws [911].

The tracking control problem of the nonholonomic mobile robots is also an important significance in project. Many researchers have focused on solving the motion control problem under nonholonomic constraints by using the kinematic model of a mobile robot $[12,13]$. These methods assume that there are some kinds of dynamic controllers that can produce perfectly the same velocity which is necessary for the kinematic controller. However,

*This paper is supported by the National Natural Science Foundation of China, No. 60874002, and the Key Project of Shanghai Education Committee, No. 09ZZ158 and the Key Project of Shanghai, No.S30501. The Fourth Phase of the Projects of Construction of upland with Electrical and Automation from Shanghai Education Committee. it is difficult to design such a dynamic controller for realization of a perfect velocity tracking. There have been a few papers where the nonholonomic kinematics controller is integrated with the dynamic model of the mobile robot $[14,15]$. Generally speaking, it is impossible to obtain the exact parameters of the kinematics and the dynamics of robots in reality. Reference [16] developed a single layer neural network for real-time motion control of a mobile robot with unknown robot dynamics and unmodeled disturbance. However, the method can't be applied to mobile robots with unknown kinematic parameters. In reference [17], an adaptive tracking controller was proposed when both dynamic and kinematic model of the mobile robot have unknown parameters, but the system controller did not consider the external disturbance. In reference [18], the external disturbance was considered, but the distance between the mass center and the geometrical center of the model of the mobile robot was known. In reference [19], the trajectory tracking control problem for the dynamic model of a nonholonomic mobile robot is discussed even in the presence of unknown parameters and bounded uncertainties. However, these methods did not consider the unknown kinematic parameters.

In this paper, a torque controller is proposed for a nonholonomic dynamic mobile robot which has a unknown distance between the mass center and the geo- 
metrical center. First, a kinematics controller is proposed to make the error between the virtual velocity and the actual velocity converge zero. Second, a torque is proposed by using backstepping method and adaptive method to make the designed velocity converge to the virtual velocity which is designed in kinematics controller previously. Using Lyapunov method, the control system is uniformly asymptotically stable. Lastly, an example is provided and the simulation results demonstrate the feasibility and efficiency of the proposed method. The comparison with the previous methods is made to show the advantages of the method in this article.

\section{Description of the Problem}

\subsection{Description of Kinematics}

We consider the mobile robot with two actuated wheels, which is shown in Figure 1. The geometrical center of the mobile robot is $C$, which is the midpoint of the two actuated wheels. The mass center of the mobile robot is $M$, and the distance from $M$ to $C$ is $d,(x, y)$ is the coordinate of $M$ in the world coordinate system $\{O, X, Y\}, \theta$ is the heading angle of the mobile robot, which is positive for anticlockwise rotation. $R$ is the distance from the geometrical center to the center of actuated wheels of robots, $r$ is the radius of the actuated wheels.

It is assumed that the mass center and the geometrical center of the robot are not coincident, which is very possible in actual situation. Assume that the wheels purely roll without slipping, the nonholonomic constraints can be expressed as:

$$
\dot{y} \cos \theta-\dot{x} \sin \theta-d \dot{\theta}=0
$$

Then the kinematic description of the robot can be expressed by:

$$
\left\{\begin{array}{l}
\dot{x}=v \cos \theta-d \omega \sin \theta \\
\dot{y}=v \sin \theta+d \omega \cos \theta \\
\dot{\theta}=\omega
\end{array}\right.
$$

where $v$ is the straight line velocity and $\omega$ is the angular velocity of the robot.

Equation (2) is represented by the matrix as follows:

$$
\dot{q}=S(q) V
$$

where

$$
\boldsymbol{S}(\boldsymbol{q})=\left[\begin{array}{cc}
\cos \theta & -d \sin \theta \\
\sin \theta & d \cos \theta \\
0 & 1
\end{array}\right], \quad \boldsymbol{V}=\left[\begin{array}{l}
v \\
\omega
\end{array}\right], \quad \boldsymbol{q}=\left[\begin{array}{l}
x \\
y \\
\theta
\end{array}\right]
$$

Taking the transformation as follows:

$$
\left\{\begin{array}{l}
Z_{1}=x \cos \theta+y \sin \theta \\
Z_{2}=-x \sin \theta+y \cos \theta
\end{array}\right.
$$

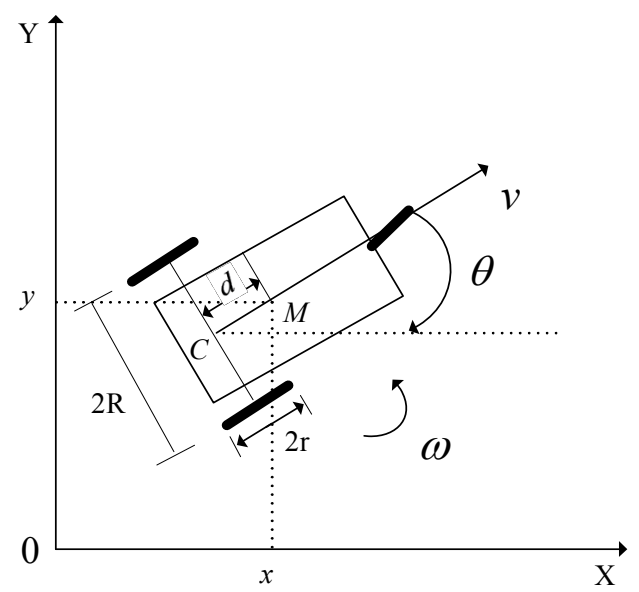

Figure 1. The model of mobile robot with two actuated wheels.

Then the kinematics description is transformed as follow:

$$
\left\{\begin{array}{l}
\dot{Z}_{1}=v-x \omega \sin \theta+y \omega \cos \theta=\omega Z_{2}+v \\
\dot{Z}_{2}=d \omega-x \omega \cos \theta-y \omega \sin \theta=-\omega Z_{1}+d \omega \\
\dot{\theta}=\omega
\end{array}\right.
$$

Because (5) is an orthogonal transformation, which doesn't change the value of modulus, it is concluded that the tracking error of original model converges to zero so long as the tracking error of the new one converges to zero. Suppose that the reference kinematics model is given as follows:

$$
\left\{\begin{array}{l}
\dot{Z}_{1 \mathrm{r}}=\omega_{\mathrm{r}} Z_{2 \mathrm{r}}+v_{\mathrm{r}} \\
\dot{Z}_{2 \mathrm{r}}=-\omega_{\mathrm{r}} Z_{1 \mathrm{r}}+d \omega_{\mathrm{r}} \\
\dot{\theta}_{\mathrm{r}}=\omega_{\mathrm{r}}
\end{array}\right.
$$

where $v_{\mathrm{r}}$ is the ideal straight line velocity and $\omega_{\mathrm{r}}$ is the ideal angular velocity of the robot.

The tracking error of system is given as follows:

$$
\mathbf{e}_{p}=\left[\begin{array}{c}
e_{1} \\
e_{2} \\
e_{3}
\end{array}\right]=\left[\begin{array}{c}
Z_{1}-Z_{1 \mathrm{r}} \\
Z_{2}-Z_{2 \mathrm{r}} \\
\theta-\theta_{\mathrm{r}}
\end{array}\right]
$$

Therefore

$$
\left\{\begin{array}{l}
\dot{e}_{1}=Z_{2} \omega-Z_{2 \mathrm{r}} \omega_{\mathrm{r}}+v-v_{\mathrm{r}} \\
\dot{e}_{2}=-Z_{1} \omega-Z_{1 \mathrm{r}} \omega_{\mathrm{r}}+d \omega-d \omega_{\mathrm{r}} \\
\dot{e}_{3}=\omega-\omega_{\mathrm{r}}
\end{array}\right.
$$

As

$$
\begin{aligned}
\dot{e}_{1} & =Z_{2} \omega-Z_{2 \mathrm{r}} \omega+Z_{2 \mathrm{r}} \omega-Z_{2 \mathrm{r}} \omega_{\mathrm{r}}+v-v_{\mathrm{r}} \\
& =e_{2} \omega+Z_{2 \mathrm{r}}\left(\omega-\omega_{\mathrm{r}}\right)+v-v_{\mathrm{r}}
\end{aligned}
$$




$$
\begin{aligned}
\dot{e}_{2} & =-Z_{1} \omega+Z_{1 \mathrm{r}} \omega-Z_{1 \mathrm{r}} \omega+Z_{1 \mathrm{r}} \omega_{\mathrm{r}}+d \omega-d \omega_{\mathrm{r}} \\
& =-e_{1} \omega-Z_{1 \mathrm{r}}\left(\omega-\omega_{\mathrm{r}}\right)+d\left(\omega-\omega_{\mathrm{r}}\right)
\end{aligned}
$$

Therefore,

$$
\left\{\begin{array}{l}
\dot{e}_{1}=e_{2} \omega+Z_{2 \mathrm{r}}\left(\omega-\omega_{\mathrm{r}}\right)+v-v_{\mathrm{r}} \\
\dot{e}_{2}=-e_{1} \omega-Z_{1 \mathrm{r}}\left(\omega-\omega_{\mathrm{r}}\right)+d\left(\omega-\omega_{\mathrm{r}}\right) \\
\dot{e}_{3}=\omega-\omega_{\mathrm{r}}
\end{array}\right.
$$

\subsection{Description of Dynamics}

When the $d$ is known, the common tracking problem is to design velocity control inputs $v$ and $\omega$ in order to make (10) asymptotically stable. However $d$ is difficult to be precisely known in fact. With $d$ is unknown, this article gives an adaptive tracking controller in the third section. In engineering practice, it is more realistic to formulate the nonholonomic control problems at dynamic levels, where the torque or force are chosen as control inputs.

The dynamics description of the robot is [14]:

$$
\boldsymbol{M}(\boldsymbol{q}) \ddot{\boldsymbol{q}}+\boldsymbol{C}(\boldsymbol{q}, \dot{\boldsymbol{q}}) \dot{\boldsymbol{q}}+\boldsymbol{F}(\dot{\mathbf{q}})+\boldsymbol{G}(\boldsymbol{q})+\tau_{\mathrm{d}}=\boldsymbol{B}(\boldsymbol{q}) \boldsymbol{\tau}-\boldsymbol{A}^{\mathrm{T}}(\boldsymbol{q}) \lambda
$$

$$
\boldsymbol{A}(\boldsymbol{q}) \dot{\boldsymbol{q}}=0
$$

where $\boldsymbol{q}$ is generalized coordinates, and $\boldsymbol{M}(\boldsymbol{q})$ is a symmetric, positive definite inertia matrix, $\boldsymbol{C}(\boldsymbol{q}, \dot{\boldsymbol{q}})$ is the centripetal and Coriolis matrix, $\boldsymbol{F}(\dot{\boldsymbol{q}})$ denotes the surface friction, $\boldsymbol{G}(\boldsymbol{q})$ is the gravitational vector, $\boldsymbol{\tau}_{\mathrm{d}}$ denotes bounded unknown disturbances including unstructured unmodelled dynamics, $\boldsymbol{B}(\boldsymbol{q})$ is the input transformation matrix, $\boldsymbol{\tau}=\left(\boldsymbol{\tau}_{1}, \boldsymbol{\tau}_{2}\right), \boldsymbol{\tau}_{1}, \boldsymbol{\tau}_{2}$ is the torque applied to the right and left wheels, $\lambda$ is the vector of constraint forces, $\boldsymbol{A}(\boldsymbol{q})$ is the matrix associated with the constraints. Considering the mobile robot under the nonholonomic constraints, we can get $\boldsymbol{A}(\boldsymbol{q})$ as follows:

$$
A(\boldsymbol{q})=\left[\begin{array}{lll}
-\sin \theta & \cos \theta & -d
\end{array}\right]
$$

From (3) and (12), we have

$$
\boldsymbol{A}(\boldsymbol{q}) \boldsymbol{S}(\boldsymbol{q})=0
$$

Differentiating both sides of (3), substituting it into (11) and pre-multiplying both sides by $\boldsymbol{S}^{\mathrm{T}}(\boldsymbol{q})$, one obtains

$$
\boldsymbol{S}^{\mathrm{T}} \boldsymbol{M S} \dot{\boldsymbol{V}}+\boldsymbol{S}^{\mathrm{T}}(\boldsymbol{M} \dot{\boldsymbol{S}}+\boldsymbol{C S}) \boldsymbol{V}+\boldsymbol{S}^{\mathrm{T}} \boldsymbol{F}+\boldsymbol{S}^{\mathrm{T}} \boldsymbol{G}+\boldsymbol{S}^{\mathrm{T}} \boldsymbol{\tau}_{\mathrm{d}}=\boldsymbol{S}^{\mathrm{T}} \boldsymbol{B} \boldsymbol{\tau}
$$

Giving $\overline{\boldsymbol{M}}=\boldsymbol{S}^{\mathrm{T}} \boldsymbol{M S}, \overline{\boldsymbol{C}}=\boldsymbol{S}^{\mathrm{T}}(\boldsymbol{M} \dot{\boldsymbol{S}}+\boldsymbol{C S})$,

$$
\overline{\boldsymbol{F}}=\boldsymbol{S}^{\mathrm{T}} \boldsymbol{F}, \overline{\boldsymbol{G}}=\boldsymbol{S}^{\mathrm{T}} \boldsymbol{G}, \overline{\boldsymbol{\tau}}_{\mathrm{d}}=\boldsymbol{S}^{\mathrm{T}} \boldsymbol{\tau}_{\mathrm{d}}, \overline{\boldsymbol{B}}=\boldsymbol{S}^{\mathrm{T}} \boldsymbol{B},
$$

then (15) can be transformed as follow:

$$
\overline{\boldsymbol{M}} \dot{\boldsymbol{V}}+\overline{\boldsymbol{C}} \boldsymbol{V}=\overline{\boldsymbol{B}} \boldsymbol{\tau}-\overline{\boldsymbol{G}}-\overline{\boldsymbol{F}}-\overline{\boldsymbol{\tau}}_{\mathrm{d}}
$$

According to [14], the matrice of dynamic Equation
(11) are given

$$
\begin{gathered}
\boldsymbol{M}(\boldsymbol{q})=\left[\begin{array}{ccc}
m & 0 & m d \sin \theta \\
0 & m & -m d \cos \theta \\
m d \sin \theta & -m d \cos \theta & I
\end{array}\right], \\
\boldsymbol{C}(\boldsymbol{q}, \dot{\boldsymbol{q}})=\left[\begin{array}{llr}
0 & 0 & m d \dot{\theta} \cos \theta \\
0 & 0 & m d \dot{\theta} \sin \theta \\
0 & 0 & 0
\end{array}\right], \boldsymbol{G}(\boldsymbol{q})=0, \\
\boldsymbol{B}(\boldsymbol{q})=\frac{1}{r}\left[\begin{array}{cc}
\cos \theta & \cos \theta \\
\sin \theta & \sin \theta \\
R & -R
\end{array}\right],
\end{gathered}
$$

therefore

$$
\begin{gathered}
\overline{\boldsymbol{M}}(\boldsymbol{q})=\left[\begin{array}{cc}
m & 0 \\
0 & I-m d^{2}
\end{array}\right], \quad \overline{\boldsymbol{C}}(\boldsymbol{q}, \dot{\boldsymbol{q}})=\left[\begin{array}{ll}
0 & 0 \\
0 & 0
\end{array}\right], \\
\\
\overline{\boldsymbol{G}}(\boldsymbol{q})=0, \overline{\boldsymbol{B}}(\boldsymbol{q})=\frac{1}{r}\left[\begin{array}{rr}
1 & 1 \\
R & -R
\end{array}\right]
\end{gathered}
$$

where $I$ is inertia moment of the robot, $m$ is mass, $R$ is the distance from the geometrical center to the center of actuated wheels of robots, $r$ is the radius of the actuated wheels.

Let $\boldsymbol{V}_{\mathrm{C}}=\left(v_{\mathrm{C}}, \omega_{\mathrm{C}}\right)^{\mathrm{T}}$ be the virtual control velocity of kinematics system (10). Assume that the velocity tracking error is

$$
\boldsymbol{\eta}=\boldsymbol{V}-\boldsymbol{V}_{C}=\left(\eta_{1}, \eta_{2}\right)^{\mathrm{T}}=\left(v-v_{C}, \omega-\omega_{C}\right)^{\mathrm{T}}
$$

Substituting (17) into (16) and using the linear property of inertial parameters of robot, we obtain

$$
\overline{\boldsymbol{M}} \dot{\boldsymbol{\eta}}+\overline{\boldsymbol{C}} \boldsymbol{\eta}=\overline{\boldsymbol{B}} \boldsymbol{\tau}-\boldsymbol{Y} \boldsymbol{\varphi}-\overline{\boldsymbol{F}}-\overline{\boldsymbol{\tau}}_{\mathrm{d}}
$$

where

$$
\boldsymbol{Y} \boldsymbol{\varphi}=\overline{\boldsymbol{M}} \dot{\boldsymbol{V}}_{\mathrm{C}}+\overline{\boldsymbol{C}} \boldsymbol{V}_{\mathrm{C}}
$$

$\varphi$ is the inertial parameter vector of robot, e.g., inertial moment and mass, $Y$ is a known matrix having nothing to do with the inertial parameter $\varphi$ of robot. By calculating, one obtains as follow:

$$
\boldsymbol{Y}=\left[\begin{array}{ccc}
\dot{\boldsymbol{v}}_{C} & 0 & 0 \\
0 & \dot{\boldsymbol{\omega}}_{c} & -\dot{\boldsymbol{\omega}}_{c}
\end{array}\right], \quad \phi=\left[\begin{array}{c}
m \\
I \\
m d^{2}
\end{array}\right]
$$

In actual motion, surface friction vector $\boldsymbol{F}(\dot{\boldsymbol{q}})$ and disturbances vector $\tau_{\mathrm{d}}$ are bounded by a known function. Therefore $\overline{\boldsymbol{F}}(\dot{\boldsymbol{q}})$ and $\overline{\boldsymbol{\tau}}_{\mathrm{d}}$ are also bounded by a known function. It could be assumed that

$$
\left\|\overline{\boldsymbol{F}}(\dot{\boldsymbol{q}})+\overline{\boldsymbol{\tau}}_{\mathrm{d}}\right\| \leq N(\boldsymbol{q}, \dot{\boldsymbol{q}})
$$

where $\boldsymbol{N}(\boldsymbol{q}, \dot{\boldsymbol{q}})$ is a known function.

The dynamic tracking problem of robots is to design a control force or a torque $\tau$ in order to make closed- 
loop system (10) and (18) be asymptotically stable. This problem will be discussed in next section.

\section{Adaptive Controller Design}

\subsection{Adaptive Kinematic Controller Design}

To make the error of equation (10) converge zero, assume that $\hat{d}$ is the estimate of $d$, and $\tilde{d}$ is the estimate error of $d$, therefore $\tilde{d}=\hat{d}-d$. Then the virtual trajectory tracking control law of the kinematic model of robot can be given as follows.

Theorem 1 Suppose that $\forall t \in[0,+\infty), Z_{1 \mathrm{r}}, Z_{2 \mathrm{r}}$ are all bounded, and the lower limit of $\left|w_{r}(h)\right|$ satisfies the condition that

$$
\lim _{t \rightarrow \infty} \inf _{h \in[t,+\infty)}\left|\omega_{\mathrm{r}}(h)\right|=a_{0}>0
$$

then if we apply the velocity control law in (21) and the adaptive control law in (22) to system (10), the kinematic tracking error described by equation (10) is asymptotically stable. And

$$
\lim _{t \rightarrow \infty}\left\|\left(e_{1}, e_{2}, e_{3}\right)^{\mathrm{T}}\right\|=0,
$$

namely the tracking error converges to zero.

$$
\boldsymbol{V}_{\mathrm{c}}=\left[\begin{array}{c}
v_{\mathrm{c}} \\
\omega_{\mathrm{c}}
\end{array}\right]=\left[\begin{array}{c}
v_{\mathrm{r}}-k_{1} e_{1} \\
\omega_{\mathrm{r}}-k_{2}\left(e_{1} Z_{2 \mathrm{r}}-e_{2} Z_{1_{\mathrm{r}}}+e_{2} \hat{d}+e_{3}\right)
\end{array}\right]
$$

The adaptive control law of $\hat{d}$ is:

$$
\dot{\hat{d}}=-\frac{1}{a}\left(k_{2} e_{1} e_{2} Z_{2 \mathrm{r}}-k_{2} e_{2}{ }^{2} Z_{1 \mathrm{r}}+k_{2} e_{2} \hat{d}+k_{2} e_{2} e_{3}\right)
$$

where $k_{1}>0, k_{2}>0, a$ is a positive constant.

The proof below needs two lemmas as follows:

Lemma 1 (Barbalat's Lemma [20]) If the differentiable function $V$ has a lower bound as $V: R^{n} \times[0, \infty) \rightarrow R$, and $\dot{V}(x, t)$ is Semi-negative Definite, and if $\dot{V}(x, t)$ is uniformly continuous about time $t$, then we have

$$
\lim _{t \rightarrow \infty} \dot{V}(x, t)=0 .
$$

Lemma 2 (Extended Barbalat's Lemma [21]) If a given differentiable function $f(x)$ from $R^{+}$to $R$ converges to some limit value when $x$ tends to infinity, and if the derivative $\dot{f}(x)$ of this function is the sum of two terms, one being uniformly continuous and the other one tending to zero when $x$ tends to infinity, then $\dot{f}(x)$ tends to zero when $x$ tends to infinity.

Proof: Consider a Lyapunov function candidate

$$
L_{1}=\frac{1}{2}\left(e_{1}^{2}+e_{2}^{2}+e_{3}^{2}\right)+\frac{1}{2} a \tilde{d}^{2}
$$

The derivative of $L_{1}$ is

$$
\begin{aligned}
\dot{L}_{1}= & e_{1} \dot{e}_{1}+e_{2} \dot{e}_{2}+e_{3} \dot{e}_{3}+a \tilde{d} \dot{\tilde{d}} \\
= & \left(e_{1} Z_{2 \mathrm{r}}-e_{2} Z_{1 \mathrm{r}}+e_{2} \hat{d}-e_{2} \tilde{d}+e_{3}\right) \\
& {\left[-k_{2}\left(e_{1} Z_{2 \mathrm{r}}-e_{2} Z_{1 \mathrm{r}}+e_{2} \hat{d}+e_{3}\right)\right]+e_{1}\left(-k_{1} e_{1}\right)+a \tilde{d} \dot{\tilde{d}} } \\
= & -k_{2}\left(e_{1} Z_{2 \mathrm{r}}-e_{2} Z_{1 \mathrm{r}}+e_{2} \hat{d}+e_{3}\right)^{2} \\
& +k_{2} e_{2} \tilde{d}\left(e_{1} Z_{2 \mathrm{r}}-e_{2} Z_{1 \mathrm{r}}+e_{2} \hat{d}+e_{3}\right)-k_{1} e_{1}^{2}+a \tilde{d} \dot{\tilde{d}} \\
= & -k_{2}\left(e_{1} Z_{2 \mathrm{r}}-e_{2} Z_{1 \mathrm{r}}+e_{2} \hat{d}+e_{3}\right)^{2}-k_{1} e_{1}^{2} \\
& +\tilde{d}\left(k_{2} e_{1} e_{2} Z_{2 \mathrm{r}}-k_{2} e_{2}{ }^{2} Z_{1 \mathrm{r}}+k_{2} e_{2} \hat{d}+k_{2} e_{2} e_{3}+a \dot{\tilde{d}}\right)
\end{aligned}
$$

Substituting (22) into (24), yields

$$
\dot{L}_{1}=-k_{2}\left(e_{1} Z_{2 \mathrm{r}}-e_{2} Z_{1 \mathrm{r}}+e_{2} \hat{d}+e_{3}\right)^{2}-k_{1} e_{1}^{2} \leq 0
$$

Clearly, $L_{1}$ is non-negative monotonically decreasing. So $L_{1}$ has the limit. According to the Lyapunov theory and Lemma 1 , we have, $e_{1} \rightarrow 0, e_{1} Z_{2 \mathrm{r}}-e_{2} Z_{1 \mathrm{r}}+e_{2} \hat{d}+e_{3}$ $\rightarrow 0$. As $Z_{2 \mathrm{r}}$ has the limit, then

$$
\lim _{t \rightarrow \infty}\left[\left(\hat{d}-Z_{1 \mathrm{r}}\right) e_{2}+e_{3}\right] \rightarrow 0
$$

As $e_{1} \rightarrow 0, \dot{e}_{1}=Z_{2} \omega-Z_{2 \mathrm{r}} \omega_{\mathrm{r}}+v-v_{\mathrm{r}}$, and $v-v_{\mathrm{r}}=v_{\mathrm{C}}-$ $v_{\mathrm{r}} \rightarrow 0, \quad v_{\mathrm{C}}-v_{\mathrm{r}}=-k_{1} e_{1} \rightarrow 0$, it can be obtained that $Z_{2} \omega$ $-Z_{2 \mathrm{r}} \omega_{r}$ is uniformly continuous. Then according to Lemma 2 , we have, $Z_{2} \omega-Z_{2 \mathrm{r}} \omega_{r} \rightarrow 0$, so $Z_{2} \omega-Z_{2 \mathrm{r}} \omega+$ $Z_{2 \mathrm{r}} \omega-Z_{2 \mathrm{r}} \omega_{r} \rightarrow 0$, that is to say, $e_{2} \omega+Z_{2 \mathrm{r}}\left(\omega-\omega_{r}\right) \rightarrow 0$. And because $Z_{2 \mathrm{r}}$ is bounded, $\omega-\omega_{\mathrm{r}}=\omega_{c}-\omega_{r}=-k_{2}\left(e_{1} Z_{2 \mathrm{r}}\right.$ $\left.-e_{2} Z_{1 \mathrm{r}}+e_{2} \hat{d}+e_{3}\right) \rightarrow 0$. It is easily obtained that $e_{2} \omega$ is uniformly continuous. So according to Lemma 2, we have $e_{2} \omega \rightarrow 0$. And because $\omega=\omega_{c}=\omega_{r}-k_{2}\left(e_{1} Z_{2 \mathrm{r}}-e_{2}\right.$ $\left.Z_{1 \mathrm{r}}+e_{2} \hat{d}+e_{3}\right) \rightarrow \omega_{r}$, we have $e_{2} \omega_{\mathrm{r}} \rightarrow 0$, according to the assumption about $\omega_{\mathrm{r}}$ in Theorem 1 . Therefore, $e_{2} \rightarrow 0$.

$\lim _{t \rightarrow \infty}\left\|\left(e_{1}, e_{2}, e_{3}\right)^{\mathrm{T}}\right\|=0$. According to (26) and the boundness of $\hat{d}-Z_{1 \mathrm{r}}$, we have, $e_{3} \rightarrow 0$. Finally, as $t \rightarrow \infty, e_{p}$ $\rightarrow 0(p=1,2,3)$, namely.

\subsection{Adaptive Dynamic Controller Design}

In Theorem 1, the kinematic velocity tracking controller is just considered. However it is very difficult to get the ideal control velocity in reality, that is to say, the error between the actual velocity and the ideal control velocity isn't equal to zero, which means $\eta \neq 0$, so the equation (21) is just the ideal kinematic velocity control. To realize the torque control, it is apparently needed to make the velocity tracking error in Equation (17) converge zero. Suppose that the $\varphi$ in this article is unknown, and $\hat{\boldsymbol{\varphi}}$ is the estimate of $\boldsymbol{\varphi}, \tilde{\boldsymbol{\varphi}}$ is the estimate error of $\boldsymbol{\varphi}$, 
therefore $\tilde{\boldsymbol{\varphi}}=\hat{\boldsymbol{\varphi}}-\boldsymbol{\varphi}$. Then the dynamic tracking control law of the robot as follows.

Theorem 2 Assume that $\forall t \in[0,+\infty), Z_{1 \mathrm{r}}, Z_{2 \mathrm{r}}$ are all bounded. Then by using the velocity control law in (27) and the adaptive control law in (28) and (29), the velocity $\boldsymbol{V}$ of the robot converges to the virtual velocity $\boldsymbol{V}_{\mathrm{c}}$ in theorem 1, which means,

$$
\lim _{t \rightarrow \infty}\left\|\left(\eta_{1}, \eta_{2}\right)^{\mathrm{T}}\right\|=0
$$

the posture tracking error $e_{p} \rightarrow 0(p=1,2,3)$.

$$
\boldsymbol{\tau}=\overline{\boldsymbol{B}}^{-1}\left(\boldsymbol{Y} \hat{\boldsymbol{\varphi}}-\left[\begin{array}{c}
e_{1} \\
e_{1} Z_{2 \mathrm{r}}-e_{2} Z_{1 \mathrm{r}}+e_{2} \hat{d}+e_{3}
\end{array}\right]-\left[\begin{array}{l}
k_{3} \eta_{1} \\
k_{4} \eta_{2}
\end{array}\right]-\operatorname{sgn}(\boldsymbol{\eta}) \cdot \boldsymbol{N}(\boldsymbol{q}, \dot{\boldsymbol{q}})\right)
$$

The adaptive control law of $\hat{\boldsymbol{\varphi}}$ is

$$
\dot{\hat{\boldsymbol{\varphi}}}=-\boldsymbol{\Gamma} \boldsymbol{Y}^{\mathrm{T}} \boldsymbol{\eta}
$$

where $k_{3}>0, k_{4}>0, \Gamma$ is a positive matrix, and they are all designed parameters.

Because $\eta \neq 0$, the corresponding adaptive control law in Equation (22) should be as follows:

$$
\dot{\hat{d}}=-\frac{1}{a}\left(k_{2} e_{1} e_{2} Z_{2 \mathrm{r}}-k_{2} e_{2}^{2} Z_{1 \mathrm{r}}+k_{2} e_{2} \hat{d}+k_{2} e_{2} e_{3}-\eta_{2} e_{2}\right)
$$

Proof: Consider a Lyapunov function candidate

$$
L=\frac{1}{2}\left(e_{2}^{1}+e_{2}^{2}+e_{3}^{2}\right)+\frac{1}{2} a \tilde{d}^{2}+\frac{1}{2} \boldsymbol{\eta}^{\mathrm{T}} \overline{\boldsymbol{M}} \boldsymbol{\eta}+\frac{1}{2} \boldsymbol{\Gamma}^{-1} \tilde{\boldsymbol{\varphi}}^{2}
$$

The derivative of $L$ is

$$
\begin{aligned}
& \dot{L}=e_{1} \dot{e}_{1}+e_{2} \dot{e}_{2}+e_{3} \dot{e}_{3}+a \tilde{d} \dot{\tilde{d}}+\boldsymbol{\eta}^{\mathrm{T}} \overline{\boldsymbol{M}} \dot{\boldsymbol{\eta}}+\frac{1}{2} \boldsymbol{\eta}^{\mathrm{T}} \dot{\overline{\boldsymbol{M}}} \boldsymbol{\eta}+\boldsymbol{\Gamma}^{-1} \tilde{\boldsymbol{\varphi}} \dot{\tilde{\boldsymbol{\varphi}}} \\
& =\left(e_{1} Z_{2 \mathrm{r}}-e_{2} Z_{1 \mathrm{r}}+e_{2} \hat{d}-e_{2} \tilde{d}+e_{3}\right)\left[-k_{2}\left(e_{1} Z_{2 \mathrm{r}}-e_{2} Z_{1 \mathrm{r}}+e_{2} \hat{d}+e_{3}\right)+\eta_{2}\right]+e_{1}\left(-k_{1} e_{1}+\eta_{1}\right)+a \tilde{d} \dot{\tilde{d}}+\boldsymbol{\eta}^{\mathrm{T}}\left(\overline{\boldsymbol{B}} \boldsymbol{\tau}-\boldsymbol{Y} \boldsymbol{\varphi}-\overline{\boldsymbol{F}}-\overline{\boldsymbol{\tau}}_{\mathrm{d}}-\overline{\boldsymbol{C}} \boldsymbol{\eta}\right) \\
& +\frac{1}{2} \boldsymbol{\eta}^{\mathrm{T}} \dot{\overline{\boldsymbol{M}}} \boldsymbol{\eta}+\boldsymbol{\Gamma}^{-1} \tilde{\boldsymbol{\varphi}} \dot{\tilde{\boldsymbol{\varphi}}}=-k_{2}\left(e_{1} Z_{2 \mathrm{r}}-e_{2} Z_{1 \mathrm{r}}+e_{2} \hat{d}+e_{3}\right)^{2}+k_{2} e_{2} \tilde{d}\left(e_{1} Z_{2 \mathrm{r}}-e_{2} Z_{1 \mathrm{r}}+e_{2} \hat{d}+e_{3}\right)-k_{1} e_{1}^{2} \\
& +\left(e_{1} Z_{2 \mathrm{r}}-e_{2} Z_{1 \mathrm{r}}+e_{2} \hat{d}+e_{3}\right) \eta_{2}-\eta_{2} e_{2} \tilde{d}+e_{1} \eta_{1}+a \tilde{d} \dot{\tilde{d}}+\boldsymbol{\eta}^{\mathrm{T}}\left(\overline{\boldsymbol{B}} \boldsymbol{\tau}-\boldsymbol{Y} \boldsymbol{\varphi}-\overline{\boldsymbol{F}}-\overline{\boldsymbol{\tau}}_{\mathrm{d}}-\overline{\boldsymbol{C}} \boldsymbol{\eta}\right)+\frac{1}{2} \boldsymbol{\eta}^{\mathrm{T}} \dot{\overline{\boldsymbol{M}}} \boldsymbol{\eta}+\boldsymbol{\Gamma}^{-1} \tilde{\boldsymbol{\varphi}} \dot{\tilde{\boldsymbol{\varphi}}} \\
& =-k_{2}\left(e_{1} Z_{2 \mathrm{r}}-e_{2} Z_{1 \mathrm{r}}+e_{2} \hat{d}+e_{3}\right)^{2}-k_{1} e_{1}^{2}+k_{2} e_{2} \tilde{d}\left(e_{1} Z_{2 \mathrm{r}}-e_{2} Z_{1 \mathrm{r}}+e_{2} \hat{d}+e_{3}\right)-\eta_{2} e_{2} \tilde{d}+\boldsymbol{\eta}^{\mathrm{T}}\left[\begin{array}{c}
e_{1} \\
e_{1} Z_{2 \mathrm{r}}-e_{2} Z_{1 \mathrm{r}}+e_{2} \hat{d}+e_{3}
\end{array}\right]+a \tilde{d} \dot{\tilde{d}} \\
& +\frac{1}{2} \boldsymbol{\eta}^{\mathrm{T}} \dot{\overline{\boldsymbol{M}}} \boldsymbol{\eta}+\boldsymbol{\Gamma}^{-1} \tilde{\boldsymbol{\varphi}} \dot{\tilde{\boldsymbol{\varphi}}}+\boldsymbol{\eta}^{\mathrm{T}}\left(\boldsymbol{Y} \hat{\boldsymbol{\varphi}}-\boldsymbol{Y} \boldsymbol{\varphi}-\left[\begin{array}{c}
e_{1} \\
e_{1} Z_{2 \mathrm{r}}-e_{2} Z_{1 \mathrm{r}}+e_{2} \hat{d}+e_{3}
\end{array}\right]-\left[\begin{array}{l}
k_{3} \eta_{1} \\
k_{4} \eta_{2}
\end{array}\right]\right. \\
& \left.-\operatorname{sgn}(\boldsymbol{\eta}) \cdot \boldsymbol{N}(\boldsymbol{q}, \dot{\boldsymbol{q}})-\overline{\boldsymbol{F}}-\overline{\boldsymbol{\tau}}_{\mathrm{d}}-\overline{\boldsymbol{C}} \boldsymbol{\eta}\right)=\dot{L}_{1}+\tilde{\boldsymbol{\varphi}} \boldsymbol{\Gamma}^{-1}\left(\dot{\tilde{\boldsymbol{\varphi}}}+\boldsymbol{\Gamma} \boldsymbol{Y}^{\mathrm{T}} \boldsymbol{\eta}\right)-k_{3} \eta_{1}^{2} \\
& -k_{4} \eta_{2}{ }^{2}+\frac{1}{2} \boldsymbol{\eta}^{\mathrm{T}}(\dot{\overline{\boldsymbol{M}}}-2 \overline{\boldsymbol{C}}) \boldsymbol{\eta}-\boldsymbol{\eta}^{\mathrm{T}}\left(\overline{\boldsymbol{F}}+\overline{\boldsymbol{\tau}}_{\mathrm{d}}\right)-\boldsymbol{\eta}^{\mathrm{T}} \operatorname{sgn}(\boldsymbol{\eta}) \cdot \boldsymbol{N}(\boldsymbol{q}, \dot{\boldsymbol{q}})
\end{aligned}
$$

Note that $\dot{\overline{\boldsymbol{M}}}-2 \overline{\boldsymbol{C}}$ is skew symmetric, so

$$
\boldsymbol{\eta}^{\mathrm{T}}(\dot{\overline{\boldsymbol{M}}}-2 \overline{\boldsymbol{C}}) \boldsymbol{\eta}=0
$$

$$
L_{2}=-\boldsymbol{\eta}^{\mathrm{T}} \operatorname{sgn}(\boldsymbol{\eta}) \cdot \boldsymbol{N}(\boldsymbol{q}, \dot{\boldsymbol{q}})-\boldsymbol{\eta}^{\mathrm{T}}\left(\overline{\boldsymbol{F}}+\overline{\boldsymbol{\tau}}_{\mathrm{d}}\right)
$$

and choose

$$
L_{2}=-\|\boldsymbol{\eta}\| \cdot \boldsymbol{N}(\boldsymbol{q}, \dot{\boldsymbol{q}})-\boldsymbol{\eta}^{\mathrm{T}}\left(\overline{\boldsymbol{F}}+\overline{\boldsymbol{\tau}}_{\mathrm{d}}\right) \leq-\|\boldsymbol{\eta}\| \cdot \boldsymbol{N}(\boldsymbol{q}, \dot{\boldsymbol{q}})+\|\boldsymbol{\eta}\| \cdot\left\|\overline{\boldsymbol{F}}+\overline{\boldsymbol{\tau}}_{\mathrm{d}}\right\|=\|\boldsymbol{\eta}\|\left(-\boldsymbol{N}(\boldsymbol{q}, \dot{\boldsymbol{q}})+\left\|\overline{\boldsymbol{F}}+\overline{\boldsymbol{\tau}}_{\mathrm{d}}\right\|\right) \leq 0
$$

According to (25), (31), (32), we have

$$
\dot{L}=\dot{L}_{1}-k_{3} \eta_{1}^{2}-k_{4} \eta_{2}^{2}+L_{2} \leq 0
$$

Clearly, $L$ is non-negative monotonically decreasing, so $L$ has the limit, according to the Lyapunov theory and Lemma 1 , we have, $\eta_{1} \rightarrow 0, \eta_{2} \rightarrow 0$, namely

$$
\lim _{t \rightarrow \infty}\left\|\left(\eta_{1}, \eta_{2}\right)^{\mathrm{T}}\right\|=0
$$

According to the expression about $\dot{L}_{1}$ in equation (25), and using the same proof method in Lemma 1, we can obtain $e_{p} \rightarrow 0(p=1,2,3)$.

\section{Simulation}

In this section, a numerical simulation is implemented to demonstrate the feasibility of the adaptive tracking controllers which are designed in the previous section. The 
mobile robot's parameters are chosen as follows [18]: $R=0.75 \mathrm{~m} ; r=0.15 \mathrm{~m} ; d=0.25 \mathrm{~m} ; a=1 ; v_{\mathrm{r}}=10 \mathrm{~m} / \mathrm{s}$; $\omega_{\mathrm{r}}=10 \mathrm{rad} / \mathrm{s} ; k_{1}=2 ; k_{2}=0.5 ; k_{3}=2 ; k_{4}=0.5$; $m=20 \mathrm{~kg} ; I=5 \mathrm{~kg} \cdot \mathrm{m}^{2} ; \varphi_{1}=0.8 ; \varphi_{2}=1.5 ; \varphi_{3}=0.3$;

The original posture of robot is $(0.1,-0.1,0.1)$, and the original velocity is $(10,10)$. Implementing the simulation with the parameters above, the simulation results are shown from Figure 2 to Figure 7.

The trajectory to tracking a circle in plane is shown in Figure 2.

The posture tracking errors of robot are shown in Figure 3.

The estimate of $d$ which is the distance from the mass center to the geometrical center of robot is shown in Figure 4.

The velocity tracking errors of robot are shown in the following Figure 5.

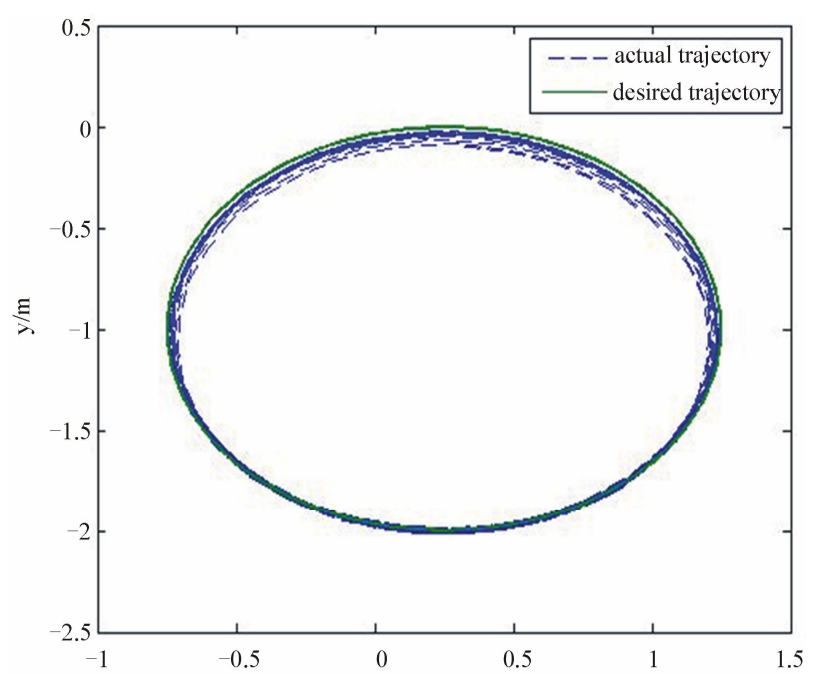

Figure 2. Tracking a circle.

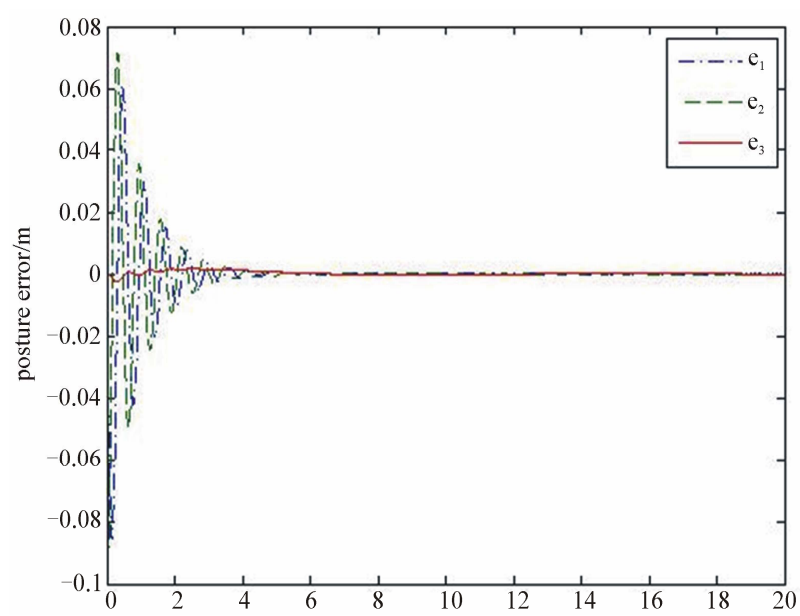

Figure 3. The posture tracking errors with respect to time.

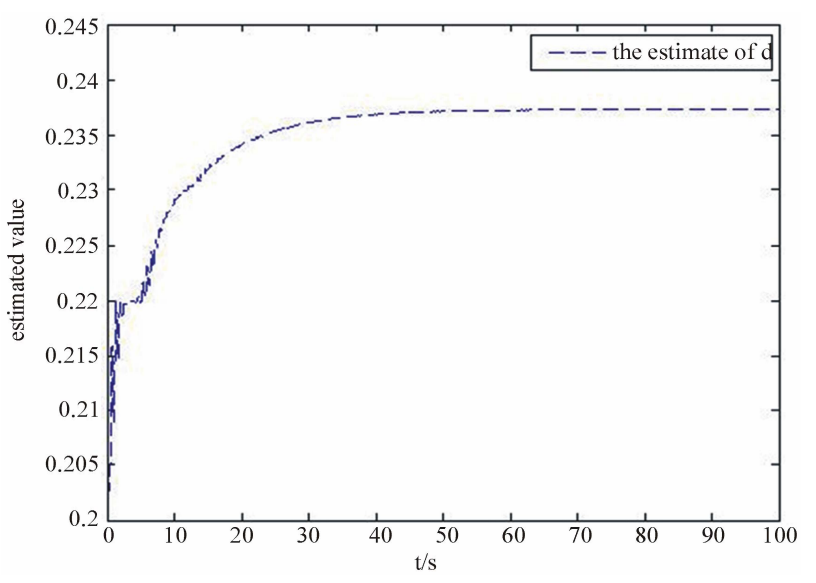

Figure 4. The estimate of $d$.

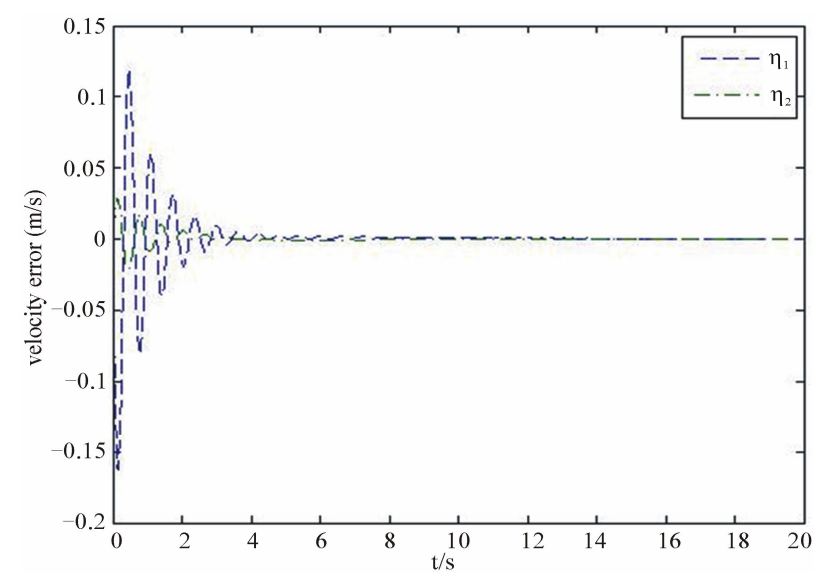

Figure 5. The velocity tracking errors with respect to time.

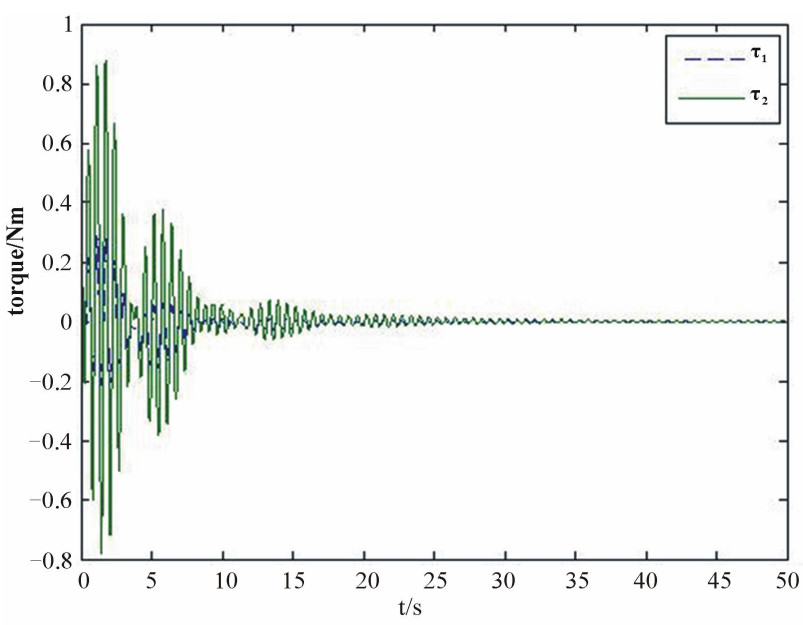

Figure 6. The torque acted on the wheels with respect to time.

The torque acted on the wheels of robot is shown in Figure 6.

The estimated dynamics parameters $\varphi_{1} 、 \varphi_{2} 、 \varphi_{3}$ are shown in Figure 7. 


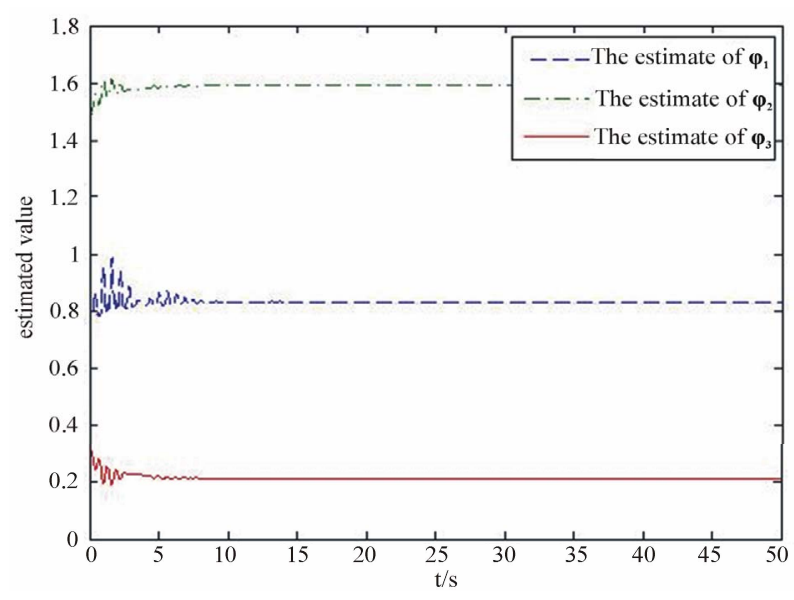

Figure 7. The estimated parameters $\varphi_{1}, \varphi_{2}, \varphi_{3}$.

We can see from Figure 2 that the robot can well track a circle. From Figures 3, 5 and $\mathbf{6}$, we can see that the posture tracking errors $e_{1}, e_{2}, e_{3}$, and velocity tracking errors $\eta_{1}, \eta_{2}$, and the torque $\tau_{1}, \tau_{2}$ acted on the wheels of robot all asymptotically converge zero. It is seen that from Figures 4 and 7 that the estimates of unknown parameters $d, \varphi_{1}, \varphi_{2}, \varphi_{3}$ of robot are all bounded. So the simulation results demonstrate the feasibility and efficiency of the proposed method in this article.

In reference [18], an adaptive tracking controller was proposed for the trajectory tracking problem when both dynamic and kinematic model of the mobile robot have unknown parameters, and the external disturbance was considered. But reference [18] assumed that the distance between the mass center and the geometrical center of the model of the mobile robot was known. However, the distance is usually unknown in actual situation. So by using the controller designed in reference [18], it can hardly track a object with the shortest time.

The controller designed in this paper consider the distance is unknown to shorten the tracking time. To show the superiority of the method in this article, the comparison with the previous methods in reference [18] is made below. The posture tracking errors in reference cite [18] is shown in the Figure 8.

The velocity tracking errors in reference [18] is shown in Figure 9.

The comparison figure of Figures $\mathbf{8}$ and $\mathbf{3}$ is shown in the Figure 10.

The comparison figure of Figures $\mathbf{9}$ and $\mathbf{5}$ is shown in Figure 11.

We can see from Figure 10 that by using the controller designed in reference [18], the time needed from original posture tracking error to the accuracy 0.01 of posture tracking error is $10 \mathrm{~s}$. While by using the controller designed in this article, it is just needed $5 \mathrm{~s}$ to achieve the same accuracy. The time has been shortened

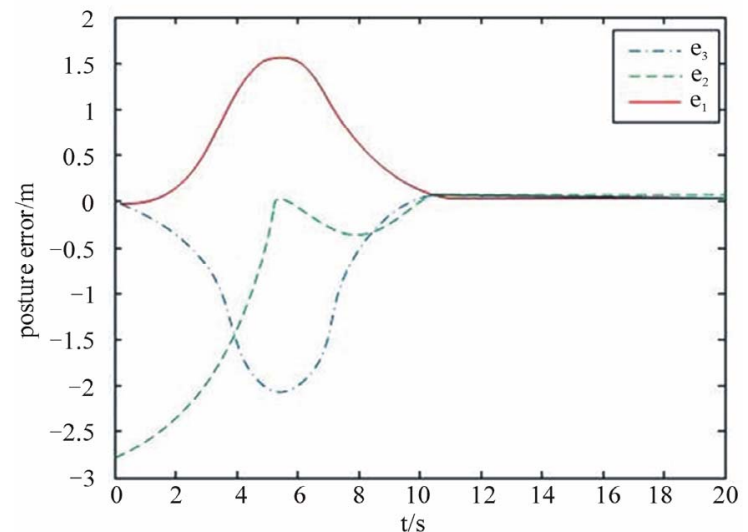

Figure 8. The posture tracking errors with respect to time in reference [18].

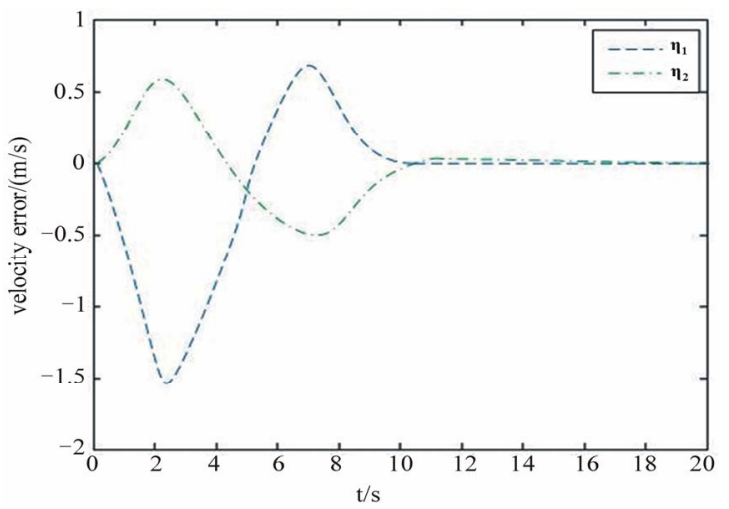

Figure 9. The velocity tracing errors with respect to time in reference [18].

by nearly one time. We can see from Figure 11 that by using the controller designed in reference [18], the time needed from original velocity tracking error to the accuracy 0.01 of velocity tracking error is $12 \mathrm{~s}$. While by using the controller designed in this article, it is just needed $5 \mathrm{~s}$ to achieve the same accuracy, the time has been shortened by more than one time. So by using the controller designed in this article, the robot can track the object more quickly. What's more, in reference [18], the distance from the mass center to the geometrical center of robot is known, while the distance in this article is unknown.

\section{Conclusions}

Based on backstepping method and adaptive control technique, a new dynamic controller for trajectory tracking problem of mobile robots with nonholonomic constraints is proposed in this article. And an adaptive feedback controller is also proposed with unknown kinematic and dynamic parameters. Using Lyapunov theory, the controller is demonstrated to be asymptoticallystable. 

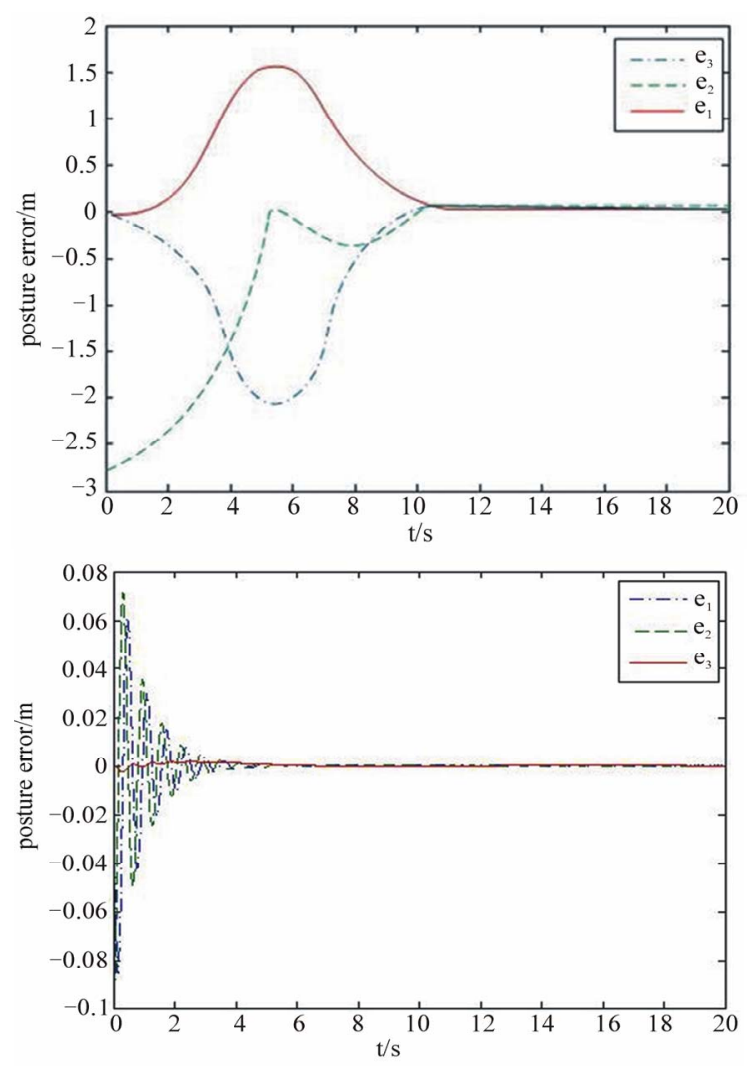

Figure 10. The comparison of Figure 8 and Figure 3.
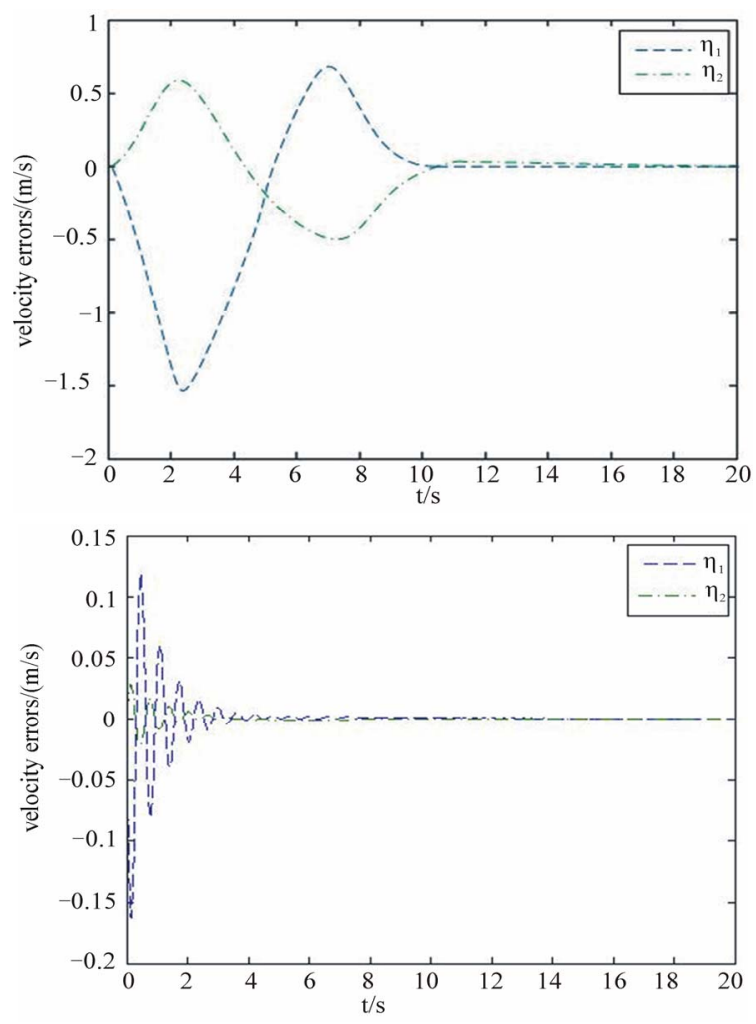

Figure 11. The comparison of Figure 9 and Figure 5.
The simulation results show the effectiveness of the proposed controller. The comparison with the previous methods is made to show the superiority of the method in this article.

\section{References}

[1] R. W. Brockett, "Differential Geometric Control Theory," Burkhauser, Boston, 1983.

[2] R. M. Murray and S. S. Sastry, "Nonholonomic Motion Planning: Steering Using Sinusoids," IEEE Transactions on Automatic Control, Vol. 38, No. 5, 1993, pp. 700-716. doi: $10.1109 / 9.277235$

[3] Y. P. Tian and S. Li, "Exponential Stabilization of Nonholonomic Dynamic Systems by Smooth Time-Varying Control," Automatica, Vol. 38, No. 7, 2002, pp. 11391146. doi:10.1016/S0005-1098(01)00303-X

[4] A. Teel, R. Murry and G. Walsh, "Nonholonomic Control Systems: From Steering To Stabilization with Sinusoids," Proceeding of 31st IEEE Conference on Decision Control, Tucson, 16-18 December 1992, pp. 1603-1609. doi:10.1109/CDC.1992.371456

[5] A. Astolfi, "Discontinuous Control of Nonholonomic Systems," Systems \& Control Letters, Vol. 27, No. 1, 1996, pp. 37-45. doi:10.1016/0167-6911(95)00041-0

[6] A. M. Bloch and S. Drakunov, "Stabilization of a Nonholonomic Systems via Sliding Modes," Proceeding of 33st IEEE Conference on Decision Control, Lake Buena Vista, 14-16 December 1994, pp. 2961-2963. doi:10.1109/CDC.1994.411342

[7] C. Canudas de Wit and O. J. Sordalen, "Exponential Stabilization of Mobile Robots with Nonholonomic Constraints," IEEE Transactions on Automatic Control, Vol. 37, No. 11, 1992, pp. 1791-1797. doi:10.1109/9.173153

[8] O. J. Sordalen and O. Egeland, "Exponential Stabilization of Nonholonomic Chained Systems," IEEE Transactions on Automatic Control, Vol. 40, No. 1, 1995, pp.35-49. doi: $10.1109 / 9.362901$

[9] P. Soueres, A. Balluchi and A. Bicchi, "Optimal Feedback Control for Line Tracking with a Bounded-Curvature Vehicle," International Journal of Control, Vol. 74, No. 10, 2001, pp. 1009-1019.

[10] I. I. Hussein and A. M. Bloch, "Optimal Control of Underactuated Nonholonomic Mechanical Systems," IEEE Transactions on Automatic Control, Vol. 53, No. 3, 2008, pp. 668-682. doi:10.1109/TAC.2008.919853

[11] Z. Qu, J. Wang, C. E. Plaisted and R. A. Hull, "GlobalStabilizing Near-Optimal Control Design for Nonholonomic Chained Systems," IEEE Transactions on Automatic Control, Vol. 51, No. 9, 2006, pp. 1440-1456. doi:10.1109/TAC.2006.880965

[12] Y. Haowen, S. Yang and G. S. Mittal, "Tracking Control of a Nonholonomic Mobile Robot by Integrating Feedback and Neural Dynamics Techniques," Proceedings of the 2003 IEEE/RSJ International Conference on Intelligent Robots and Systems, Miami, 27-31 October 2003, pp. 
3522- 3527. doi:10.1109/IROS.2003.1249701

[13] Y. Kanayama, Y. Kimura and T. Noguchi, "A Stable Tracking Method for an Autonomous Mobile Robot," Proceedings of 1990 IEEE International Conference on Robotics and Automation, Cincinnati, 13-18 May 1990, pp. 384-389. doi: 10.1109/ROBOT.1990.126006

[14] R. Fierro and F. L. Lewis, "Control of a Nonholonomic Mobile Robot: Backstepping Kinematics into Dynamics," Proceeding of the 34th Conference on Decision and Control, New Orleans, 13-15 December 1995, pp. 3805-3810. doi: 10.1109/CDC.1995.479190

[15] J. Yang and J. Kim, "Sliding Mode Motion Control of Nonholonomic Mobile Robots," IEEE Control Systems, Vol. 19, No. 2, 1999, pp. 15-23. doi: $10.1109 / 37.753931$

[16] T. Hu and S. X. Yang, "An Efficient Neural Controller for a Nonholonomic Mobile Robot," Proceedings of 2001 IEEE International Conference on Robotics and Automation, Piscataway, 2001, pp. 461-466. doi:10.1109/CIRA.2001.1013245
[17] T. Fukao, H. Nakagawa and N. Adachi, "Adaptive Tracking Control of a Nonholonomic Mobile Robot," IEEE Transaction on Robotics and Automation, Vol. 16, No. 5, 2000, pp. 609-615. doi:10.1109/70.880812

[18] J. B. Wu, G. H. Xu and Z. P. Yin, "Robust Adaptive Control for a Nonholonomic Mobile Robot with Unknown Parameters," Journal of Control Theory and Applications, Vol. 7, No. 2, 2009, 212-218. doi:10.1007/s11768-009-7130-6

[19] M. Yan, Q. Wu and Y. He, "Adaptive Sliding Mode Tracking Control of Nonholonomic Mobile Robot," Journal of System Simulation, Vol. 19, No. 3, 2007, pp. 579-581.

[20] J. J. E. Slotine and W. P. Li, "Applied Nonlinear Control," Prentice Hall, Upper Saddle River, 1991.

[21] C. Samson, "Control of Chained Systems Application to Path Following and Time-Varying Point-Stabilization of Mobile Robots," IEEE Transactions on Automatic Control, Vol. 40, No. 1, 1995, pp. 64-77. doi:10.1109/9.362899 